\title{
CLDN1 regulates trophoblast apoptosis and proliferation in preeclampsia
}

\author{
Yu-chen Zhang 1,2, Xiao-li Qin², Xiao-ling Ma², Hui-qin Mo ${ }^{3}$, Shi Qin ${ }^{3}$, Cheng-xi Zhang ${ }^{1,2}$, \\ Xiao-wei Wei ${ }^{1,2}$, Xue-qing Liu' ${ }^{1,2}$, Yan Zhang ${ }^{4}$, Fu-ju Tian ${ }^{1,2}$ and Yi Lin ${ }^{1,2}$ \\ ${ }^{1}$ Shanghai Key Laboratory of Embryo Original Diseases, The International Peace Maternity \& Child Health Hospital, \\ Shanghai Jiao Tong University School of Medicine, Shanghai, China, ${ }^{2}$ The International Peace Maternity \& Child \\ Health Hospital, Shanghai Jiao Tong University School of Medicine, Shanghai, China, ${ }^{3}$ Department of Obstetrics \\ and Gynecology, Shanghai First Maternity and Infant Hospital, Tongji University of Medicine, Shanghai, China and \\ ${ }^{4}$ Department of Obstetrics and Gynecology, Renmin Hospital of Wuhan University, Wuhan, Hu Bei, China
}

Correspondence should be addressed to Y Zhang or F Tian or Y Lin; Email: zyan2200@gmail.com or fjtian@sibs.ac.cn oryilinonline@126.com

\begin{abstract}
Preeclampsia is a gestational hypertensive disease; however, preeclampsia remains poorly understood. Bioinformatics analysis was applied to find novel genes involved in the pathogenesis of preeclampsia and identified CLDN1 as one of the most differentially expressed genes when comparing patients with preeclampsia and healthy controls. The results of the qRT-PCR, Western blotting and immunohistochemistry experiments demonstrated that CLDN1 was significantly downregulated in the chorionic villi in samples from patients with preeclampsia. Furthermore, knockdown of CLDN1 in HTR-8/SVneo cells resulted in the inhibition of proliferation and induction of apoptosis, and overexpression of CLDN1 reversed these effects. In addition, RNA-seq assays demonstrated that the gene $B I R C 3$ is potentially downstream of CLDN1 and is involved in the regulation of apoptosis. Knockdown of CLDN1 confirmed that the expression level of BIRC3 was obviously decreased and was associated with a significant increase in cleaved PARP. Interestingly, the apoptotic effect in CLDN1 knockdown cells was rescued after BIRC3 overexpression. Overall, these results indicate that a decrease in CLDN1 inhibits BIRC3 expression and increases cleaved PARP levels thus participating in the pathogenesis of preeclampsia.
\end{abstract}

Reproduction (2021) 161 623-632

\section{Introduction}

Preeclampsia (PE) is a complication of pregnancy characterised by high blood pressure, and it leads to a series of unpleasant outcomes, such as placental abruption, intrauterine growth retardation and preterm birth (Mol et al. 2016). According to the 2019 ACOG Practice Bulletin, PE is diagnosed based on abnormal blood pressure after 20 weeks of gestation with nonobligatory confirmation by the presence of proteinuria (ACOG 2019). PE can be accompanied by multiorgan dysfunction, including thrombocytopenia, renal insufficiency, impaired liver function and nervous system dysfunction. To date, many studies have investigated the mechanism of this gestational hypertensive disorder; however, detailed molecular mechanism of the disease is unknown. A widely accepted theory describes the pathogenesis of the disease as a 'two stage' process: abnormal placentation occurring in the first-trimester of pregnancy and consequent maternal syndrome in the second- or third-trimester of pregnancy. Under physiological circumstances, endovascular extravillous trophoblast entered inside spiral arteries vessel wall after interstitial extravillous trophoblast invasion; shallow invasion of endovascular extravillous trophoblast results in insufficient conversion of spiral arteries and the consequent poor placentation (Turco \& Moffett 2019, Staff et al. 2020). Insufficient trophoblast invasion results in incomplete spiral artery remodelling and consequent reduced placental perfusion, and decidual vasculopathy and poor uterine decidualisation are potential reasons leading to placental insufficiency (Fisher 2015, Phipps et al. 2019, Rana et al. 2019). Increasing evidence has demonstrated that both foetal and maternal factors contribute to abnormal placentation. The development of technologies and numerous tactics assisting with the exploration of the mechanism of the disease can be used to identify additional contributing factors.

Claudin-1 is encoded by the CLDN1 gene and is the first member of the claudin family. CLDN1 is a component of the tight junction complex, which controls epithelial homeostasis by regulating paracellular movement of the molecules (Garcia-Hernandez 
et al. 2017). Dysfunction of CLDN1 is involved in the pathogenesis of multiple diseases. For example, CLDN1deficient mice die soon after birth due to cutaneous dehydration (Furuse et al. 2002). Patients with atopic dermatitis show reduced expression of CLDN1 in the nonlesional epidermis (De Benedetto et al. 2011). A recent study demonstrated that CLDN1 plays a key role in cutaneous wound healing (Volksdorf et al. 2017). In addition, CLDN1 is widely expressed in the intestinal epithelium; increased expression of CLDN1 is observed in intestinal inflammatory disorders, such as Crohn's disease and ulcerative colitis (Garcia-Hernandez et al. 2017). Abnormal expression levels of CLDN1 were also detected in malignant tumours (Stache et al. 2014, Huang et al. 2015, Zhang et al. 2016). Diminished expression of CLDN1 has been detected in PE placentas in other studies (Lievano et al. 2006, Pirinen \& Soini 2014); however, the mechanism behind this phenotype has not been demonstrated.

In the current study, we confirmed that the expression of CLDN1 was downregulated in the placental villus tissue in PE compared to that in uncomplicated pregnancy and identified a novel function of CLDN1 in trophoblasts. Moreover, inhibition of CLDN1 expression results in an increase in cleaved PARP via downregulation of $B I R C 3$ expression.

\section{Materials and methods GEO data analysis}

GEO datasets (GSE66273, GSE75010, GSE96984 and GSE114691) were downloaded from the GEO database (https://www.ncbi.nlm.nih.gov/geo/). PCA was initially used to evaluate the expression profiles between the two groups (Supplementary Fig. 1, see section on supplementary materials given at the end of this article), and the expression matrix was validated prior to subsequent analysis. Differential genes based on the array data were analysed using the limma package for R. For RNA-seq data, transcripts were filtered and preprocessed using the voom function before differential gene identification using the limma package. Genes with adjusted $P$ values $<0.05$ ( $\mathrm{BH}$ method) and absolute $\log _{2} \mathrm{FC}>1$ were identified as differentially expressed genes.

\section{Patient information}

Last trimester placental villus tissue was collected at the International Peace Maternity \& Child Health Hospital, China Welfare Institute, Shanghai Jiao Tong University School of Medicine. Patients with the following diagnoses were excluded: (1) foetal chromosomal malformation; (2) pregnancy complicated with endocrine or metabolic diseases (e.g. gestational diabetes mellitus, hypothyroidism and hyperthyroidism); (3) chorioamnionitis; (4) deleterious physical or chemical exposure during gestation; and (5) other diseases associated with pregnancy complications (e.g. previously diagnosed hypertension and renal diseases). A total of 25 preeclampsia (PE) patients and 27 subjects with uncomplicated pregnancies at 32-41 gestational weeks were included based on the diagnostic criteria for PE of the 2019 ACOG Practice Bulletin (Table 1 and Supplementary Table 2). All patients were fully informed, and consent was obtained in a written form. Ethics approval for this study was granted by the Medical Ethics Committee of the International Peace Maternity \& Child Health Hospital of the China Welfare Institute, Shanghai, China.

\section{RNA-seq and downstream bioinformatics analysis}

HTR-8/SVneo cells were transfected with siRNA or negative control for $48 \mathrm{~h}$ as described above. Total RNA of three paired samples of HTR-8/SVneo cells was reserved using $1 \mathrm{~mL}$ TRIzol (Ambion) and then transferred to Novogene (Beijing). Total RNA was extracted and RNA quality was measured using an Agilent 2100 bioanalyzer. The transcriptome library was constructed by a NEBNext ${ }^{\circledR}$ Ultra $^{\text {TM }}$ RNA library prep kit for Illumina ${ }^{\circledR}$; and sequencing was performed on the Illumina Novaseq platform. After quality control, reads were mapped to reference genome using Hisat2. DEseq2 package for $\mathrm{R}$ was used for normalisation and to identify differentially expressed genes. Genes with adjusted $P$ values less than 0.05 were used to perform enrichment analysis using the ClusterProfiler package for R (Yu et al. 2012).

\section{Statistical analysis}

All data were acquired from at least three independent biological replicates. Data were presented as the mean \pm S.D., and statistical analysis was performed using GraphPad Prism 8. For parametric data, a Student's $t$-test was used to compare two groups. For nonparametric data, a Mann-Whitney test was used to compare two groups or a Kruskal-Wallis test was used to compare three or more groups. $P$ values $<0.05$ were considered statistically significant.

Additional materials and methods are provided in Supplementary Information.

Table 1 Clinical information of patients included in this study. Data were presented as the mean \pm S.D. and statistical analysis was done performed using GraphPad Prism 8. A Student's t-test or Mann-Whitney test was used to compare two groups.

\begin{tabular}{lcc}
\hline Characteristics & $\begin{array}{c}\text { Healthy controls } \\
(n=27)\end{array}$ & $\begin{array}{c}\text { Preeclampsia } \\
(n=25)\end{array}$ \\
\hline Maternal age, years & $30.19 \pm 3.076$ & $33.52 \pm 4.656^{* * *}$ \\
Gestational day & $273.3 \pm 12.14$ & $256.1 \pm 16.70^{* * * *}$ \\
Systolic blood pressure, & $117.1 \pm 9.698$ & $150.5 \pm 15.12^{* * * *}$ \\
$\quad$ mmHg & $72.41 \pm 6.128$ & $97.48 \pm 8.809^{* * * *}$ \\
Diastolic blood pressure, & & \\
mmHg & $3144 \pm 470.3$ & $2620 \pm 703.3^{* *}$ \\
Infant weight, g & $600.2 \pm 105.1$ & $553.4 \pm 162.1^{* *}$ \\
\hline Placenta weight, g & &
\end{tabular}

**P $<0.01$, **** $P<0.0001$. 


\section{Results}

\section{CLDN1 was downregulated in the placental villi of patients with preeclampsia}

To determine whether certain genes are involved in the pathogenesis of PE, four GEO datasets of the placental transcriptome in preeclampsia (PE) and healthy controls (HCs) were selected (Leavey et al. 2016, Than et al. 2018, Awamleh et al. 2019). Interestingly, two genes were identified based on the intersection of differentially expressed genes of the datasets, including CLDN1 and BHLHE4O (Fig. 1A). QRT-PCR was performed to confirm the results of bioinformatics analysis, and the level of CLDN1 was found to be significantly decreased in the placental villi of PE patients compared to that in HCs (Fig. 1B); however, the level of BHLHE4O was not significantly different in the present qRT-PCR result; therefore, we chose CLDN1 for further investigation. Western blotting and immunohistochemical staining
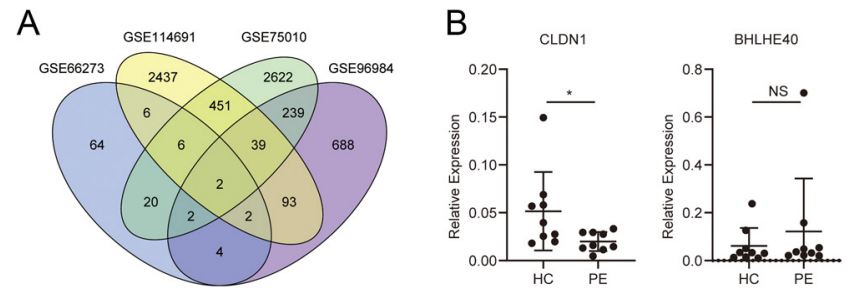

C
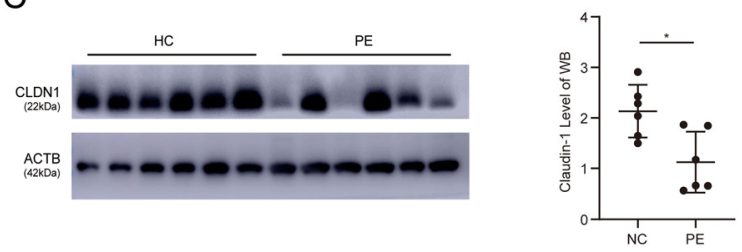

D
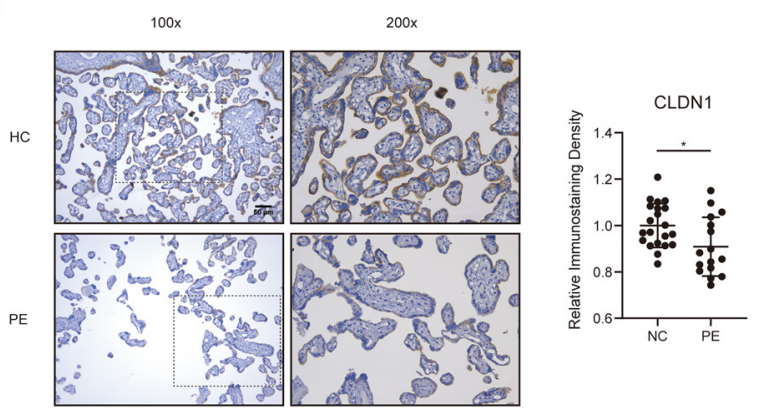

Figure 1 CLDN1 is decreased in the placental villus tissue of patients with preeclampsia. (A) Bioinformatics analysis of differentially expressed genes from the GEO datasets. (B) CLDN1 expression in placental villi from preeclampsia (PE) patients or healthy controls (HCs) was determined using qRT-PCR $(n=9)$. (C) Western blotting $(n=6)$ of the expression of CLDN1 in preeclampsia patients. (D) Images of IHC staining for CLDN1 ( $n=21$ in the HC group, $n=16$ in the PE group) and relative immunostaining density scores in the PE and $\mathrm{HC}$ groups quantified using Image-Pro Plus 6.0. A

Mann-Whitney test was used in (B), and a Student's $t$-test was used in (C) and (D), and the data are presented as the mean \pm S.D. ${ }^{*} P<0.05$, NS, not significant. were used to confirm a decrease in the expression of CLDN1 in the placental villus tissue in PE (Fig. 1C and D). Thus, CLDN1 expression is decreased in trophoblasts of PE patients, suggesting that this decrease may be involved in trophoblast dysfunction.

\section{Knockdown of CLDN1 inhibits proliferation and induces apoptosis in trophoblasts}

To investigate the role of CLDN1 in PE, we determined the expression level of CLDN1 in three commonly used trophoblast cell lines, HTR-8/SVneo, JAR and Bewo, using gRT-PCR and Western blotting. The results showed that HTR-8/SVneo had the highest level of CLDN1 expression (Fig. 2A), and immunofluorescence result showed the subcellular localisation of CLDN1 in HTR-8/SVneo (Fig. 2B). Therefore, we selected HTR-8/ SVneo cells as an in vitro model to study the function of CLDN1 in trophoblasts. We used siRNA to knock down CLDN1 in HTR-8/SVneo cells, and knockdown efficiency was measured by Western blotting (Fig. 2C). Furthermore, the results of the CCK-8 assay indicated that CLDN1 knockdown significantly inhibited the proliferation of trophoblasts (Fig. 2D). Accordingly, the EdU assay demonstrated that knockdown of CLDN1 resulted in a decrease in the proportion of EdU-positive cells, which confirmed that downregulation of CLDN1 impairs trophoblast proliferation (Fig. 2E). The effect of CLDN1 on trophoblast apoptosis was evaluated by flow cytometry, and the results showed that knockdown of CLDN1 promoted trophoblast apoptosis at both early and late stages (Fig. 2F). TUNEL staining confirmed that CLDN1 knockdown induced apoptosis of the cells (Fig. 2G).

\section{Overexpression of CLDN1 promotes proliferation and alleviates apoptosis in trophoblasts}

We used a CLDN1-overexpressing plasmid to investigate the role of CLDN1 in trophoblasts. The overexpression efficiency was determined by Western blotting (Fig. $3 \mathrm{~A})$. The results of the CCK-8 assay demonstrated an increase in cell viability after CLDN1 overexpression (Fig. 3B), and the data of the EdU assay also indicated that CLDN1 overexpression promoted cell proliferation (Fig. 3C). Furthermore, the results of flow cytometry showed that CLDN1 overexpression decreased the proportion of apoptosis mainly in the late stage (Fig. 3D). Correspondingly, the data of the TUNEL assay showed that CLDN1 overexpression reduced apoptosis in trophoblasts though not significant (Fig. 3E).

\section{CLDN1 downregulation affects apoptosis-associated pathways}

Since CLDN1 has a significant influence on HTR-8/ SVneo apoptosis, we tested apoptosis associated 


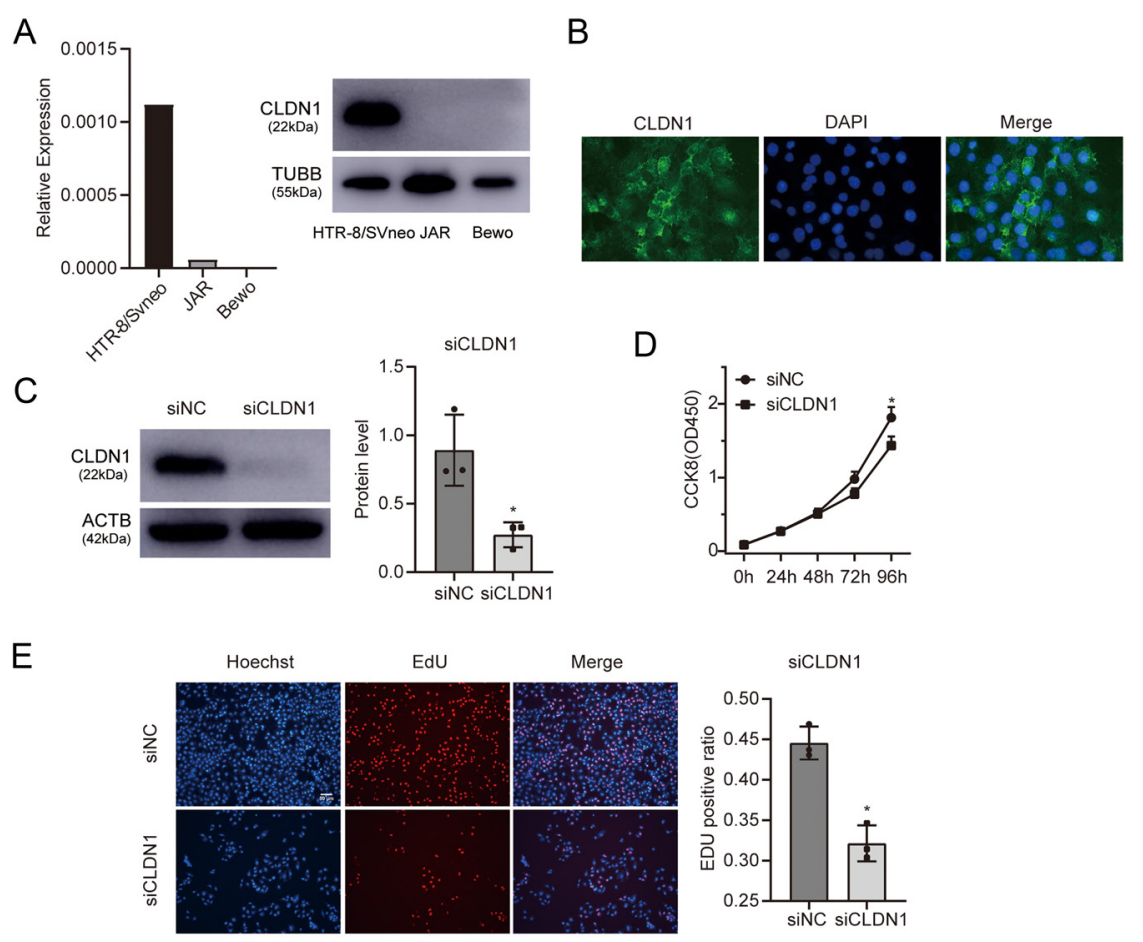

$\mathrm{F}$
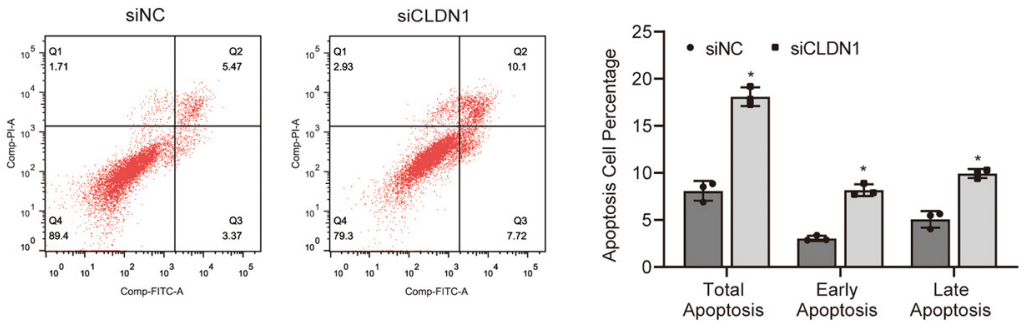

G
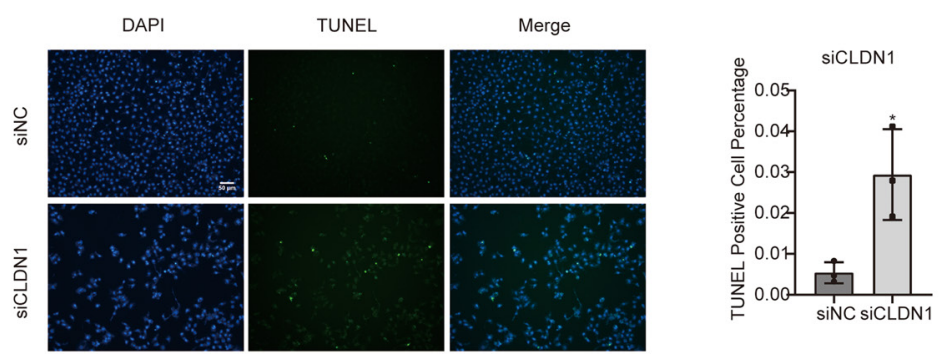

Figure 2 Knockdown of CLDN1 inhibits the proliferation and induces apoptosis in trophoblasts. (A) CLDN1 expression levels in three commonly used trophoblast cell lines. (B) Subcellular localisation of CLDN1 in HTR-8/SVneo cell line. (C) Expression of CLDN1 after siCLDN1 oligonucleotide administration. (D) CCK-8 assay showing trophoblast proliferation at indicated time points. (E) EdU assay showing the role of CLDN1 in cell proliferation. (F) Apoptosis was assessed by flow cytometry analysis. (G) TUNEL assay verifying an increase in the proportion of apoptotic cells after CLDN1 downregulation. Each in vitro test test was used, and the data are presented as the mean \pm S.D. ${ }^{*} P<0.05 ; \mathrm{NS}$, not significant. protein BAX and BCL2 in CLDN1 downregulated cells. However, the results showed that CLDN1 may not affect apoptosis through these two proteins (Supplementary Fig. 2). RNA-seq assay was used to determine the molecular mechanism of regulation of trophoblast proliferation and apoptosis by CLDN1; we identified the differences in the transcriptomes of HTR-8/SVneo cells treated with negative control siRNA (siNC) or siRNA against CLDN1. A total of 268 differentially expressed genes were identified, including 144 upregulated and 124 downregulated genes (Fig. 4A and B). Genes with adjusted $P$ values less than 0.05 were used to perform the enrichment analysis (Fig. 4C and D). For KEGG analysis, significantly enriched terms included protein processing in endoplasmic reticulum, ubiquitin-mediated proteolysis, apoptosis and cell cycle. Furthermore, qRTPCR was used to validate the RNA sequencing results of the top 13 differentially expressed genes (Fig. 4E). BIRC3 was selected for the downstream analysis since it was one of the most prominently differentially expressed genes enriched in the apoptosis pathway. BIRC3 encodes cellular inhibitor of apoptosis protein 2 (cIAP2), a member of the inhibitors of apoptosis protein family (IAPs), which has been reported to indirectly inhibit caspase activity (Bai et al. 2014). Therefore, we detected $B I R C 3$ protein levels in CLDN1-manipulated cells, and the results showed that $B I R C 3$ was downregulated in CLDN1 knockdown cells and upregulated in CLDN1- 
A
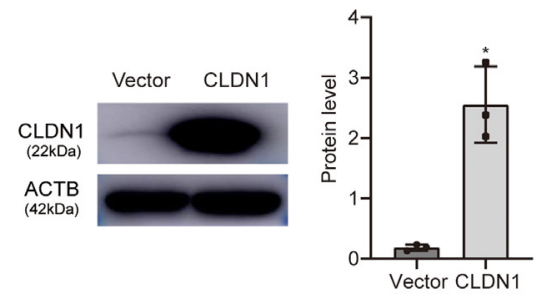

C

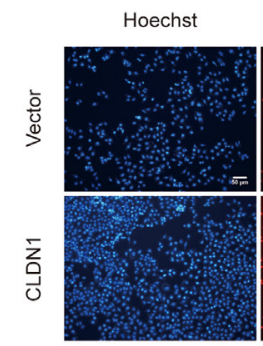

D

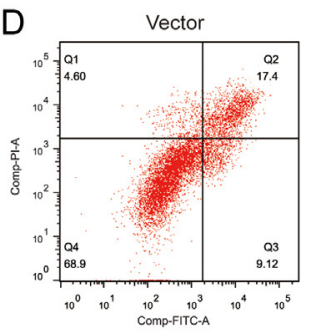

E

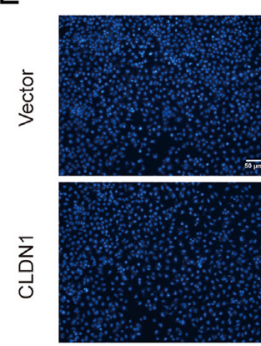

DAPI
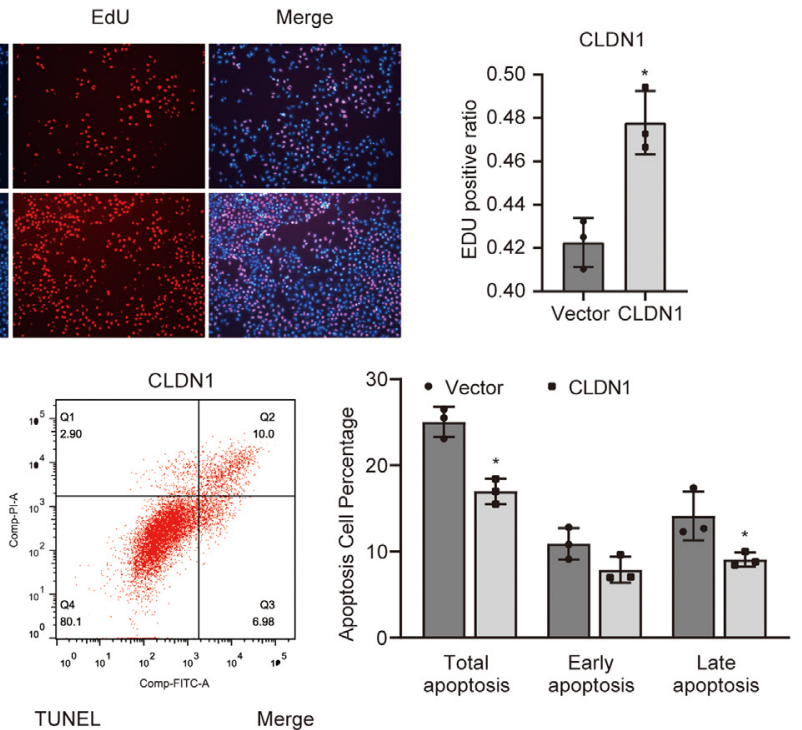

TUNEL
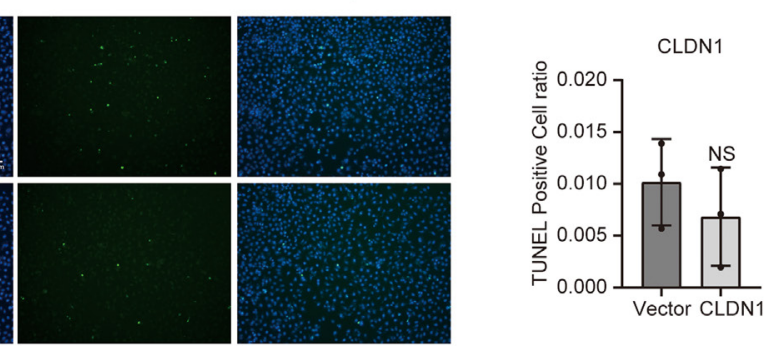

B

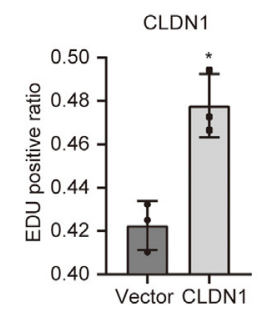

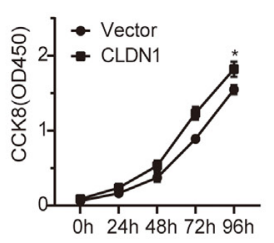

overexpressing cells (Fig. 4F). These data indicate that $C L D N 1$ regulates the expression of $B I R C 3$ in trophoblasts.

\section{CLDN1 regulates apoptosis via BIRC3 expression}

Decreased levels of $B I R C 3$ are commonly accompanied by increased levels of cleaved PARP in apoptotic cells (Zhang et al. 2019). Several small molecule mimetics of second mitochondria-derived activator of caspases (SMAC) have been developed to restore apoptosis in the tumour cells (Bai et al. 2014, Wang et al. 2016). Western blotting was performed to determine whether PARP is involved in trophoblast apoptosis, and the results showed that cleaved PARP was elevated by CLDN1 downregulation, and the overexpression of CLDN1 decreased the level of cleaved PARP (Fig. $5 A)$. To verify that the CLDN1/BIRC3/PARP pathway regulates apoptosis of HTR-8/SVneo cells, we used flow cytometry to analyse the effect of $B I R C 3$ overexpression on apoptosis after CLDN1 knockdown (Fig. 5B).
The results showed that apoptosis was successfully reduced after $B I R C 3$ overexpression. In addition, cleaved PARP levels were decreased after overexpression of BIRC3 in CLDN1 knockdown HTR-8/SVneo cells (Fig. $5 \mathrm{C})$. Furthermore, BIRC3 expression was measured in $\mathrm{PE}$ and $\mathrm{HC}$ villus tissue using Western blotting, and we found that BIRC3 is decreased in the PE placentas compared with $\mathrm{HC}$ placentas though the difference is not significant (Fig. 5D). Thus, our results suggested that CLDN1 inhibited BIRC3 mRNA levels and that downstream PARP participated in trophoblast apoptosis.

\section{Discussion}

Accumulating studies have demonstrated the transcriptome differences between placenta in preeclampsia (PE) and uncomplicated pregnancy (Nishizawa et al. 2007, Awamleh et al. 2019). In contrast, this study combined four GEO datasets to compare the transcriptome of placenta in preeclampsia and normal 
A

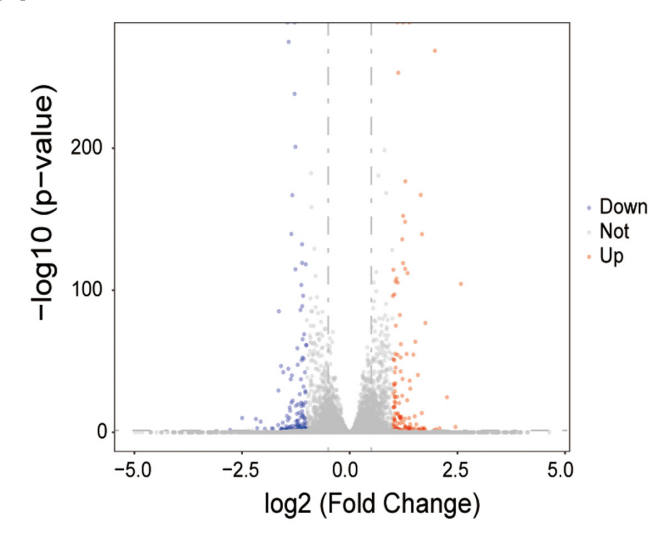

C

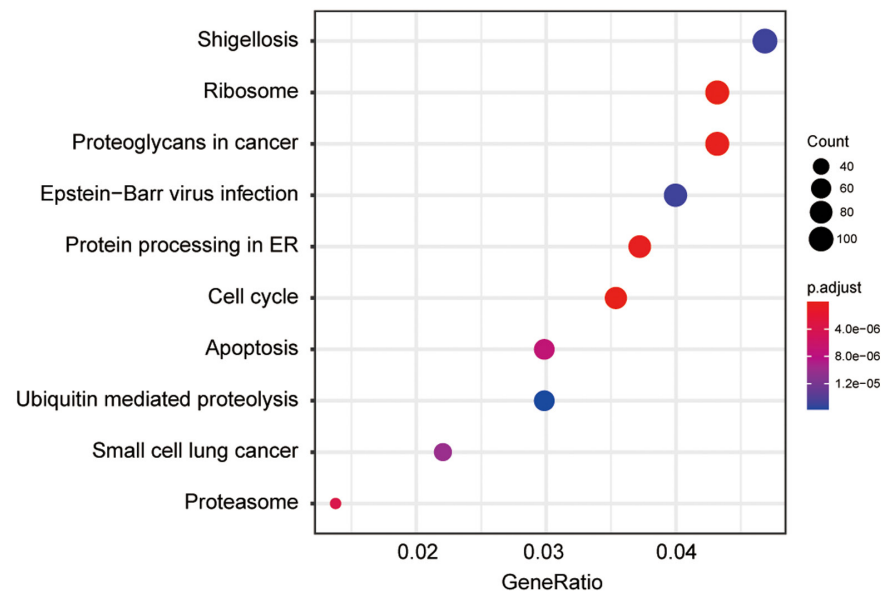

D

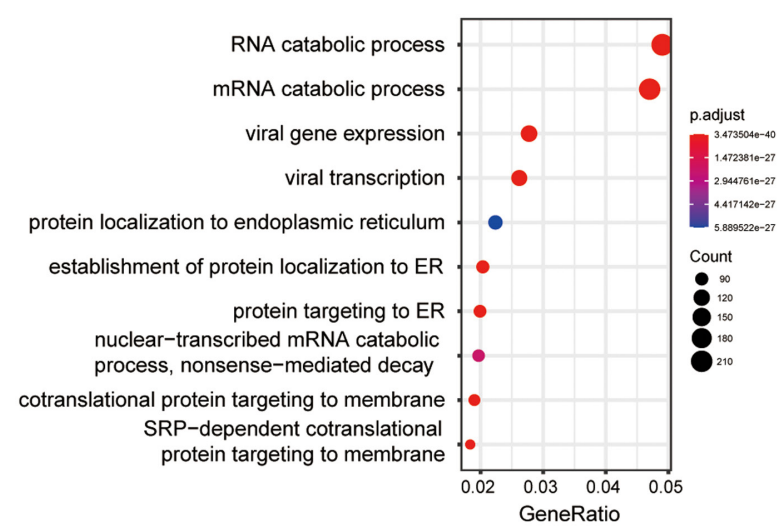

B

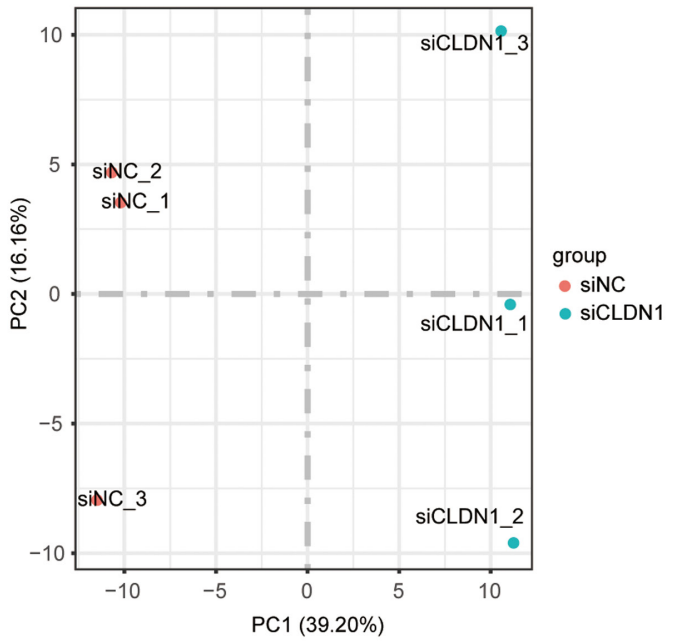

E

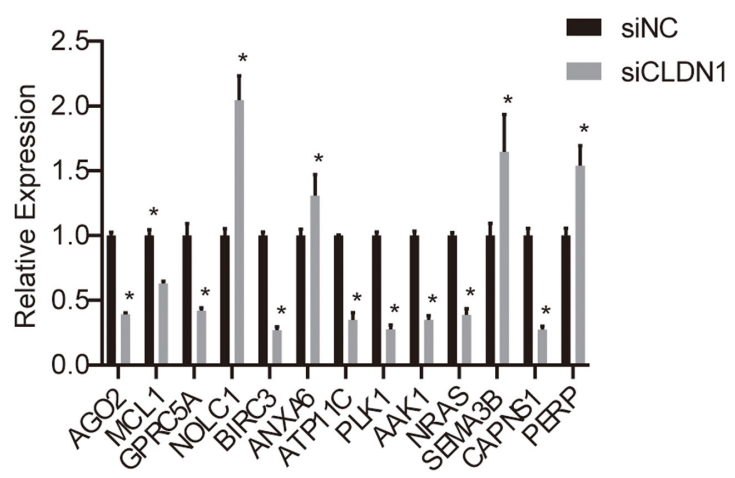

F
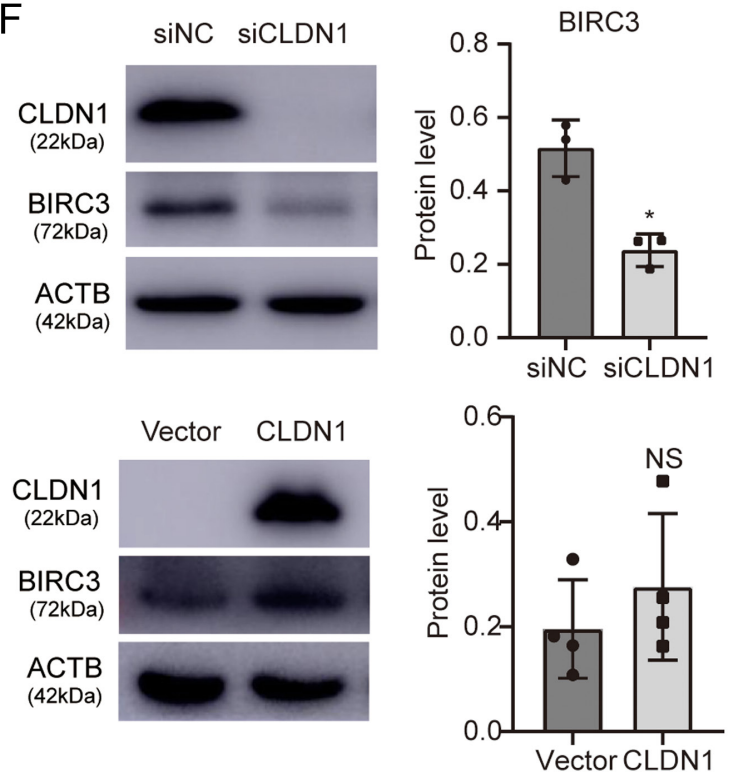

Figure 4 CLDN1 downregulation affects apoptosis-associated pathways. (A and B) Volcano and heatmap plots showing differentially expressed genes after CLDN1 knockdown. (C and D) KEGG and GO enrichment analysis. (E) qRT-PCR results of differentially expressed genes after CLDN1 downregulation. (F) Western blotting assay of BIRC3 after CLDN1 downregulation or overexpression. Each in vitro test was performed three times, Mann Whitney test was used, and the data are presented as the mean \pm S.D. ${ }^{*} P<0.05$, NS, not significant. 
A
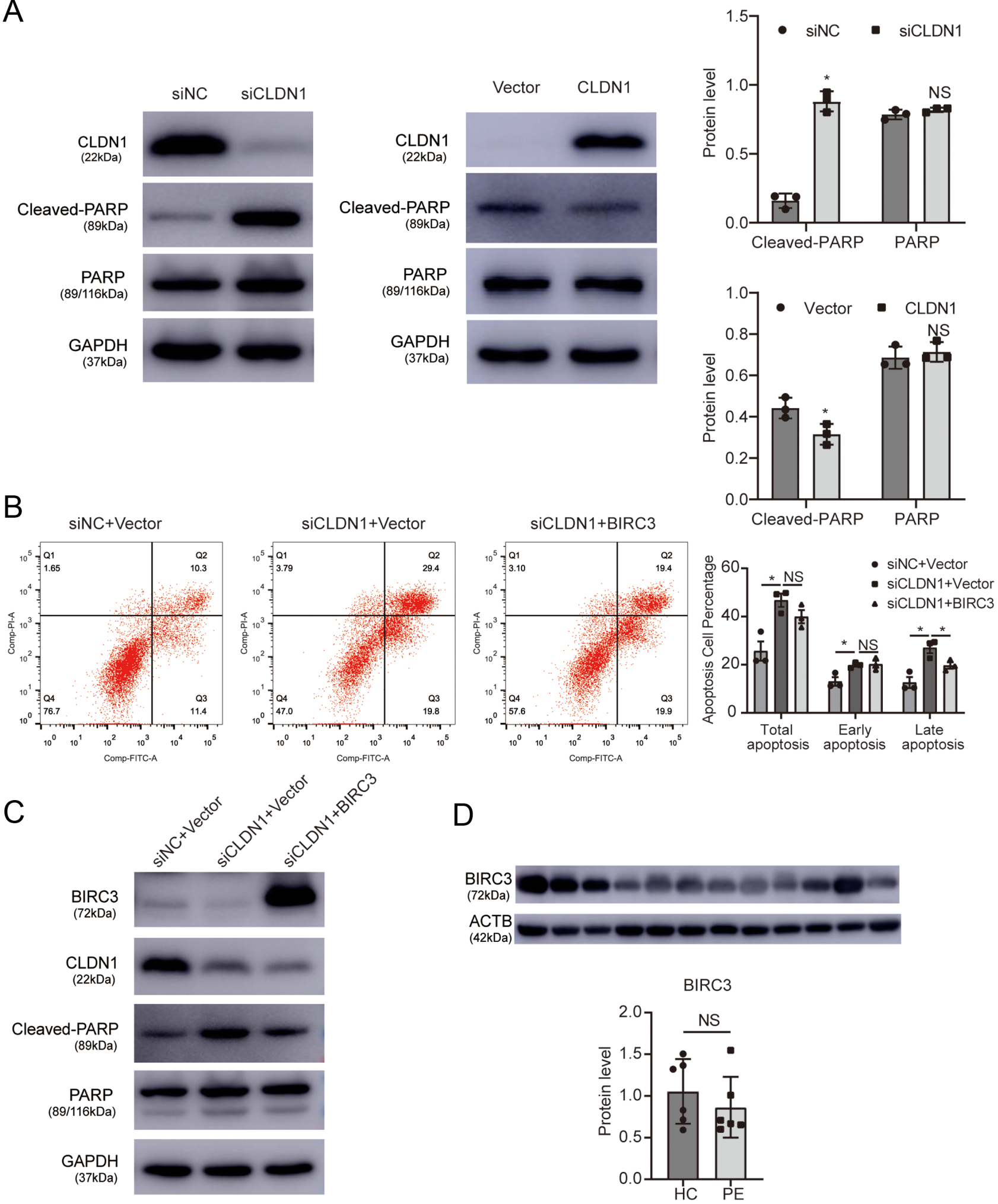

Figure 5 CLDN1 regulates apoptosis through the BIRC3/PARP pathway. (A) Western blotting showing alteration in the levels of cleaved PARP after CLDN1 downregulation or overexpression. (B) BIRC3 overexpression in CLDN1-downregulated cells reduced trophoblasts apoptosis according to the data of flow cytometry. (C) Level of cleaved PARP after BIRC3 overexpression in CLDN1-downregulated cells. (D) Protein level of BIRC3 in HC and PE. Each in vitro test was performed three times, Mann Whitney test were used in (A) and (D) and Kruskal-Wallis test was used in (B), and the data are presented as the mean \pm S.D. ${ }^{*} P<0.05, \mathrm{NS}$, not significant. 
pregnancy using bioinformatics analysis to demonstrate potential differential expression of the CLDN1 gene. Both the mRNA and protein levels of CLDN1 were decreased in the placental villi tissue of preeclampsia patients. The tight junction complex plays an important role in cellular activities including proliferation, migration and so on (Garcia-Hernandez et al. 2017). As an important protein regulating cell-cell attachment, we assume that the downregulation of CLDN1 to some extent disrupted the completeness of plasma membrane which is vital to maintain the integrity of the cell function. In this article, we confirmed that a decrease in CLDN1 leads to the induction of apoptosis in trophoblasts.

Increased apoptosis of villus trophoblasts in placenta pathologies has been observed in many pregnancy-associated diseases, including pregnancy loss, preeclampsia and intrauterine growth restriction (Ishihara et al. 2002, Sharp et al. 2010). Hypoxiareoxygenation and oxidative stress are associated with trophoblast apoptosis (Soleymanlou et al. 2007). Moreover, Kalkat et al. reported that the balance of BCL2 family proteins was altered towards cell death in preeclampsia (Kalkat et al. 2013). Recent studies have focused on trophoblast apoptosis caused by molecular dysfunction that leads to the disease (Guo et al. 2017, Wang \& Yan 2018, Mo et al. 2020). CLDN1 has been shown to be involved in apoptosis via alterations in the expression levels of Bcl-2 and Bax (Singh et al. 2012, Zheng et al. 2019), which was not detected in our study (Supplementary Fig. 2). We suggest that this difference may be due to the heterogeneity of tissue origin.

The impact of the CLDN1 gene on apoptosis has been investigated in a number of studies. For example, CLDN1 downregulation was shown to promote apoptosis and hinder cell proliferation in tumour cells (Huang et al. 2015, Zheng et al. 2019). Some studies demonstrated that CLDN1 was associated with the activation of autophagy (Zhao et al. 2017), and other studies concluded that CLDN1 regulates anoikis (Singh et al. 2012, Huang et al. 2015). Our data indicated that downregulation of CLDN1 inhibited the proliferation and induced apoptosis in trophoblasts. Since the detailed mechanism by which CLDN1 regulates trophoblast apoptosis is unclear, we used RNA-seq in CLDN1knockdown HTR-8/SVneo cells. The results identified $B I R C 3$ as a molecule downstream of CLDN1. BIRC3 was shown to be involved in the regulation of both intrinsic and extrinsic apoptosis pathways via indirect regulation of caspase activity (Varfolomeev et al. 2007, Bai et al. 2014, Gressot et al. 2017, Lee et al. 2017, Zhang et al. 2019). Majority of claudins contain a PDZ binding motif that interacts with intercellular molecules that have signalling functions (Garcia-Hernandez et al. 2017). As a tight junction protein, claudin-1 was confirmed to be associated with $\mathrm{Src} / \mathrm{p}-\mathrm{Src}$ in colon cancer cells in modulating cell anoikis (Singh et al. 2012). It was also found that claudin-1 regulates cell anoikis through
ZEB-1 (Singh et al. 2011). Evidence has also been found that claudin-1 regulates apoptosis in human breast cancer cells through deactivation of pro-caspase- 8 (Liu et al. 2012). Other claudin family member such as claudin-8 interacts with IL22 in colon cell lines to rescue apoptosis induced by LINC00662 knockdown (Cheng et al. 2020). However, the mechanism of how CLDN1 regulates downstream BIRC3 remains to be determined.

Accumulated cleaved PARP has been detected in other trophoblast cell lines in a study that demonstrated that increased apoptosis in trophoblasts is accompanied by an increase in the levels of proapoptotic proteins (Chen et al. 2015). We hypothesised that the accumulation of cleaved PARP caused by BIRC3 downregulation may be involved in the mechanism of PE pathogenesis. Thus, we investigated the effect of CLDN1 knockdown on the level of cleaved PARP. The results showed that knockdown of CLDN1 increased the level of cleaved PARP, and subsequent experiments showed that overexpression of BIRC3 in CLDN1-downregulated HTR-8/SVneo cells reduced cell apoptosis. Some studies showed associations between BIRC3 and necrosis (Geserick et al. 2009, Bai et al. 2014); hence, we examined the impact of CLDN1 downregulation on trophoblast necrosis. However, the levels of phosphorylated RIP and phosphorylated MLKL showed trends that were not consistent with this assumption (Supplementary Fig. 2).

In conclusion, we demonstrated that downregulation of CLDN1 regulates trophoblast apoptosis via a decrease in the levels of BIRC3 and downstream PARP, which is a new phenomenon in the pathogenesis of preeclampsia (Fig. 6). Our study demonstrated the association of CLDN1 and preeclampsia, which can offer a new potential therapeutic target for future clinical applications. However, current results are based on experiments of trophoblast cell lines which is still quite different to the normal trophoblasts; and further investigation using primary trophoblast cells may help to further verify our conclusion.

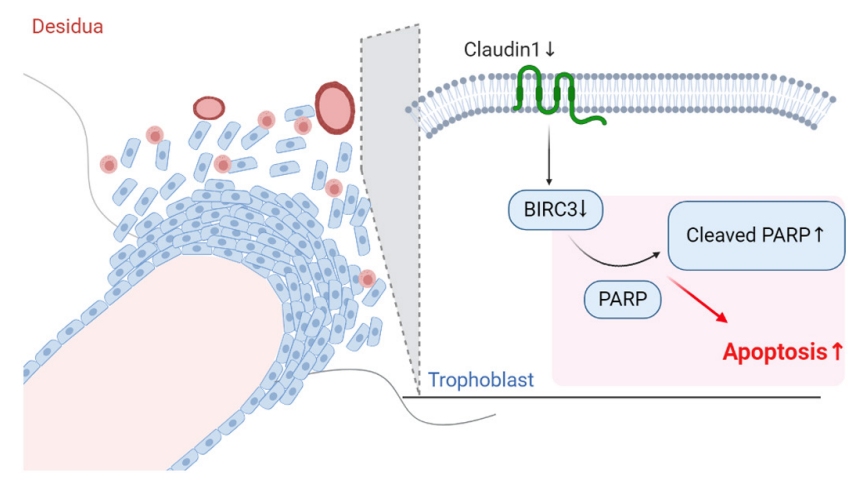

Figure 6 Schematic depiction of CLDN1 in trophoblast apoptosis. (Created with BioRender.com). 


\section{Supplementary materials}

This is linked to the online version of the paper at https://doi. org/10.1530/REP-20-0677.

\section{Declaration of interest}

The authors declare that there is no conflict of interest that could be perceived as prejudicing the impartiality of the research reported.

\section{Funding}

This work was supported by the National Key Research and Development Program of China (2018YFC1002800 to Yan Zhang and Yi Lin), the Special Funds for Local Science and Technology Development Guided by the Central Committee (2018ZYYD014 to Yan Zhang), the National Natural Science Foundation of China (81971403 to Yi Lin), the National Natural Science Foundation of China (82071647 to Fuju Tian), the National Natural Science Foundation of China (81671519 to Fengtao Shi) and the Clinical Research Fund of the International Peace Maternity and Child Health Hospital, Shanghai Jiao Tong University School of Medicine (GFY5816 to Yi Lin). The authors would like to thank the Department of Biobank (International Peace Maternity and Child Health Hospital, Shanghai Jiao Tong University) for providing clinical samples.

\section{Author contribution statement}

$\mathrm{Y}-\mathrm{C} \mathrm{Z}$ and $\mathrm{F}-\mathrm{J} \mathrm{T}$ conceived and designed the experiments. $\mathrm{Y}-\mathrm{C}$ Z, X-L Q, H-Q M, C-X Z helped to collect samples, Y-C Z, H-Q $M, S Q, X-W W$ and $X-Q L$ helped to perform the experiments. $\mathrm{Y}-\mathrm{C} Z$ and $\mathrm{F}-\mathrm{J} T$ analysed the data and wrote the manuscript, F-J $T, Y Z$ and $Y L$ contributed reagents and materials and to revise the manuscript.

\section{References}

ACOG 2019 Gestational hypertension and preeclampsia. Obstetrics and Gynecology 133 e1-e25. (https://doi.org/10.1097/ AOG.0000000000003018)

Awamleh Z, Gloor GB \& Han VKM 2019 Placental microRNAs in pregnancies with early onset intrauterine growth restriction and preeclampsia: potential impact on gene expression and pathophysiology. BMC Medical Genomics 12 91. (https://doi.org/10.1186/s12920-0190548-x)

Bai L, Smith DC \& Wang S 2014 Small-molecule SMAC mimetics as new cancer therapeutics. Pharmacology and Therapeutics 144 82-95. (https://doi.org/10.1016/j.pharmthera.2014.05.007)

Chen H, Meng T, Liu X, Sun M, Tong C, Liu J, Wang H \& Du J 2015 Long non-coding RNA MALAT-1 is downregulated in preeclampsia and regulates proliferation, apoptosis, migration and invasion of JEG3 trophoblast cells. International Journal of Clinical and Experimental Pathology 8 12718-12727.

Cheng B, Rong A, Zhou Q \& Li W 2020 LncRNA LINC00662 promotes colon cancer tumor growth and metastasis by competitively binding with miR-340-5p to regulate CLDN8/IL22 co-expression and activating ERK signaling pathway. Journal of Experimental and Clinical Cancer Research 39 5. (https://doi.org/10.1186/s13046-019-1510-7)
De Benedetto A, Rafaels NM, Mcgirt LY, Ivanov AI, Georas SN, Cheadle C, Berger AE, Zhang K, Vidyasagar S, Yoshida T et al. 2011 Tight junction defects in patients with atopic dermatitis. Journal of Allergy and Clinical Immunology 127 773.e1-786.e1. (https://doi.org/10.1016/j. jaci.2010.10.018)

Fisher SJ 2015 Why is placentation abnormal in preeclampsia? American Journal of Obstetrics and Gynecology 213 (Supplement) S115-S122. (https://doi.org/10.1016/j.ajog.2015.08.042)

Furuse M, Hata M, Furuse K, Yoshida Y, Haratake A, Sugitani Y, Noda T, Kubo A \& Tsukita S 2002 Claudin-based tight junctions are crucial for the mammalian epidermal barrier: a lesson from claudin-1-deficient mice. Journal of Cell Biology 156 1099-1111. (https://doi.org/10.1083/ jcb.200110122)

Garcia-Hernandez V, Quiros M \& Nusrat A 2017 Intestinal epithelial claudins: expression and regulation in homeostasis and inflammation. Annals of the New York Academy of Sciences 1397 66-79. (https://doi. org/10.1111/nyas.13360)

Geserick P, Hupe M, Moulin M, Wong WW, Feoktistova M, Kellert B, Gollnick H, Silke J \& Leverkus M 2009 Cellular IAPs inhibit a cryptic CD95-induced cell death by limiting RIP1 kinase recruitment. Journal of Cell Biology 187 1037-1054. (https://doi.org/10.1083/jcb.200904158)

Gressot LV, Doucette T, Yang YH, Fuller GN, Manyam G, Rao A, Latha K \& Rao G 2017 Analysis of the inhibitors of apoptosis identifies BIRC3 as a facilitator of malignant progression in glioma. Oncotarget 8 12695-12704. (https://doi.org/10.18632/oncotarget.8657)

Guo M, Zhao X, Yuan X \& Li P 2017 Elevated microRNA-34a contributes to trophoblast cell apoptosis in preeclampsia by targeting BCL-2. Journal of Human Hypertension 31 815-820. (https://doi.org/10.1038/ jhh.2017.65)

Huang J, Zhang L, He CY, Qu Y, Li JF, Zhang JN, Du T, Chen X, Yu YY, Liu BY et al. 2015 Claudin-1 enhances tumor proliferation and metastasis by regulating cell anoikis in gastric cancer. Oncotarget 6 1652-1665. (https://doi.org/10.18632/oncotarget.2936)

Ishihara N, Matsuo H, Murakoshi H, Laoag-Fernandez JB, Samoto T \& Maruo T 2002 Increased apoptosis in the syncytiotrophoblast in human term placentas complicated by either preeclampsia or intrauterine growth retardation. American Journal of Obstetrics and Gynecology 186 158-166. (https://doi.org/10.1067/mob.2002.119176)

Kalkat M, Garcia J, Ebrahimi J, Melland-Smith M, Todros T, Post M \& Caniggia I 2013 Placental autophagy regulation by the BOKMCL1 rheostat. Autophagy 9 2140-2153. (https://doi.org/10.4161/ auto.26452)

Leavey K, Benton SJ, Grynspan D, Kingdom JC, Bainbridge SA \& Cox BJ 2016 Unsupervised placental gene expression profiling identifies clinically relevant subclasses of human preeclampsia. Hypertension $\mathbf{6 8}$ 137-147. (https://doi.org/10.1161/HYPERTENSIONAHA.116.07293)

Lee JY, Tokumoto M, Hwang GW, Lee MY \& Satoh M 2017 Identification of ARNT-regulated BIRC3 as the target factor in cadmium renal toxicity. Scientific Reports 7 17287. (https://doi.org/10.1038/s41598017-17494-9)

Lievano S, Alarcon L, Chavez-Munguia B \& Gonzalez-Mariscal L 2006 Endothelia of term human placentae display diminished expression of tight junction proteins during preeclampsia. Cell and Tissue Research 324 433-448. (https://doi.org/10.1007/s00441-005-0135-7)

Liu Y, Wang L, Lin XY, Wang J, Yu JH, Miao Y \& Wang EH 2012 Antiapoptotic effect of claudin-1 on TNF-alpha-induced apoptosis in human breast cancer MCF-7 cells. Tumour Biology 33 2307-2315. (https://doi. org/10.1007/s13277-012-0493-1)

Mo HQ, Tian FJ, Ma XL, Zhang YC, Zhang CX, Zeng WH, Zhang Y \& Lin Y 2020 PDIA3 regulates trophoblast apoptosis and proliferation in preeclampsia via the MDM2/p53 pathway. Reproduction 160 293-305. (https://doi.org/10.1530/REP-20-0156)

Mol BWJ, Roberts CT, Thangaratinam S, Magee LA, De Groot CJM \& Hofmeyr GJ 2016 Pre-eclampsia. Lancet 387 999-1011. (https://doi. org/10.1016/S0140-6736(15)00070-7)

Nishizawa H, Pryor-Koishi K, Kato T, Kowa H, Kurahashi H \& Udagawa Y 2007 Microarray analysis of differentially expressed fetal genes in placental tissue derived from early and late onset severe pre-eclampsia. Placenta 28 487-497. (https://doi.org/10.1016/j.placenta.2006.05.010)

Phipps EA, Thadhani R, Benzing T \& Karumanchi SA 2019 Pre-eclampsia: pathogenesis, novel diagnostics and therapies. Nature Reviews: Nephrology 15 275-289. (https://doi.org/10.1038/s41581-019-0119-6) 
Pirinen E \& Soini Y 2014 A survey of zeb1, twist and claudin 1 and 4 expression during placental development and disease. APMIS 122 530-538. (https://doi.org/10.1111/apm.12187)

Rana S, Lemoine E, Granger JP \& Karumanchi SA 2019 Preeclampsia: pathophysiology, challenges, and perspectives. Circulation Research 124 1094-1112. (https://doi.org/10.1161/CIRCRESAHA.118.313276)

Sharp AN, Heazell AE, Crocker IP \& Mor G 2010 Placental apoptosis in health and disease. American Journal of Reproductive Immunology 64 159-169. (https://doi.org/10.1111/j.1600-0897.2010.00837.x)

Singh AB, Sharma A, Smith JJ, Krishnan M, Chen X, Eschrich S, Washington MK, Yeatman TJ, Beauchamp RD \& Dhawan P 2011 Claudin-1 up-regulates the repressor ZEB-1 to inhibit E-cadherin expression in colon cancer cells. Gastroenterology $1412140-2153$. (https://doi.org/10.1053/j.gastro.2011.08.038)

Singh AB, Sharma A \& Dhawan P 2012 Claudin-1 expression confers resistance to anoikis in colon cancer cells in a Src-dependent manner. Carcinogenesis 33 2538-2547. (https://doi.org/10.1093/carcin/bgs275)

Soleymanlou N, Jurisicova A, Wu Y, Chijiiwa M, Ray JE, Detmar J, Todros T, Zamudio S, Post M \& Caniggia I 2007 Hypoxic switch in mitochondrial myeloid cell leukemia factor-1/Mtd apoptotic rheostat contributes to human trophoblast cell death in preeclampsia. American Journal of Pathology 171 496-506. (https://doi.org/10.2353/ajpath.2007.070094)

Stache C, Holsken A, Fahlbusch R, Flitsch J, Schlaffer SM, Buchfelder M \& Buslei R 2014 Tight junction protein claudin-1 is differentially expressed in craniopharyngioma subtypes and indicates invasive tumor growth. Neuro-Oncology 16 256-264. (https://doi.org/10.1093/neuonc/not195)

Staff AC, Fjeldstad HE, Fosheim IK, Moe K, Turowski G, Johnsen GM, Alnaes-Katjavivi P \& Sugulle M 2020 Failure of physiological transformation and spiral artery atherosis: their roles in preeclampsia. American Journal of Obstetrics and Gynecology In Press. (https://doi. org/10.1016/j.ajog.2020.09.026)

Than NG, Romero R, Tarca AL, Kekesi KA, Xu Y, Xu Z, Juhasz K, Bhatti G, Leavitt RJ, Gelencser Z et al. 2018 Integrated systems biology approach identifies novel maternal and placental pathways of preeclampsia. Frontiers in Immunology 9 1661. (https://doi.org/10.3389/ fimmu.2018.01661)

Turco MY \& Moffett A 2019 Development of the human placenta. Development 146 dev163428. (https://doi.org/10.1242/dev.163428)

Varfolomeev E, Blankenship JW, Wayson SM, Fedorova AV, Kayagaki N, Garg P, Zobel K, Dynek JN, Elliott LO, Wallweber HJ et al. 2007 IAP antagonists induce autoubiquitination of C-IAPs, NF-kappaB activation, and TNFalpha-dependent apoptosis. Cell 131 669-681. (https://doi. org/10.1016/j.cell.2007.10.030)
Volksdorf T, Heilmann J, Eming SA, Schawjinski K, Zorn-Kruppa M, Ueck C, vidal-y-sy S, Windhorst S, Jucker M, Moll I et al. 2017 Tight junction proteins Claudin-1 and occludin are important for cutaneous wound healing. American Journal of Pathology 187 1301-1312. (https:// doi.org/10.1016/j.ajpath.2017.02.006)

Wang F \& Yan J 2018 MicroRNA-454 is involved in regulating trophoblast cell proliferation, apoptosis, and invasion in preeclampsia by modulating the expression of ephrin receptor B4. Biomedicine and Pharmacotherapy 107 746-753. (https://doi.org/10.1016/j. biopha.2018.08.055)

Wang DP, Berglund A, Kenchappa RS, Forsyth PA, mulé JJ \& etame AB 2016 BIRC3 is a novel driver of therapeutic resistance in Glioblastoma. Scientific Reports 6 21710. (https://doi.org/10.1038/srep21710)

Yu G, Wang LG, Han Y \& He QY 2012 clusterProfiler: an R package for comparing biological themes among gene clusters. Omics 16 284-287. (https://doi.org/10.1089/omi.2011.0118)

Zhang WN, Li W, Wang XL, Hu Z, Zhu D, Ding WC, Liu D, Li KZ, Ma D \& Wang H 2016 CLDN1 expression in cervical cancer cells is related to tumor invasion and metastasis. Oncotarget 7 87449-87461. (https://doi. org/10.18632/oncotarget.13871)

Zhang S, Yang Y, Weng W, Guo B, Cai G, Ma Y \& Cai S 2019 Fusobacterium nucleatum promotes chemoresistance to 5 -fluorouracil by upregulation of BIRC3 expression in colorectal cancer. Journal of Experimental and Clinical Cancer Research 38 14. (https://doi.org/10.1186/s13046-0180985-y)

Zhao Z, Li J, Jiang Y, Xu W, Li X \& Jing W 2017 CLDN1 increases drug resistance of non-small cell lung cancer by activating autophagy via up-regulation of ULK1 phosphorylation. Medical Science Monitor 23 2906-2916. (https://doi.org/10.12659/msm.904177)

Zheng YF, Luo J, Gan GL \& Li W 2019 Overexpression of microRNA-98 inhibits cell proliferation and promotes cell apoptosis via claudin-1 in human colorectal carcinoma. Journal of Cellular Biochemistry $\mathbf{1 2 0}$ 6090-6105. (https://doi.org/10.1002/jcb.27895)

Received 4 January 2021

First decision 19 January 2021

Revised Manuscript received 18 March 2021

Accepted 30 March 2021 